\title{
TRIBO DE CONSUMO DE ANIMES: O ANIME COMO UM TOTEM
}

\author{
TRIBE OF ANIME CONSUMPTION: \\ ANIME AS A TOTEM
}

Bruno Alves Catão ${ }^{1}$

Claudia Rosa Acevedo ${ }^{2}$

Eduardo Correa de Godoy ${ }^{3}$

Recebido em: 02 de janeiro de 2017

Aprovado em: 07 de maio de 2017

Sistema de Avaliação: Double Blind Review

RGD | v. 14 | n. 2 |p. 126-140|jul./dez. 2017

\begin{abstract}
RESUMO
O presente estudo é uma análise etnográfica das tribos de animes, com o objetivo de desvendar os valores, as práticas de consumo, a organização, o comportamento e a cultura da tribo. Utilizamos registros de vídeo, fotos e anotações de campo para coletar as informações da pesquisa de observação participante. Concluímos que os animes influenciam os estilos visuais, hábitos e padrões de consumo dos integrantes. O anime é o centro da atenção dos jovens. É uma espécie de "totem" da tribo de consumo. É devido ao consumo do anime que a tribo se forma. Os jovens pertencem ao anime e os anime lhes pertence. Isso ocorre de maneira que eles se tornam algo dentro de um todo, fortalecendo as suas identidades e conferindo sentido às suas vidas. $\mathrm{O}$ consumo dentro da tribo possui forte dimensão simbólica. Possuir produtos culturais relacionados aos animes contribui para a própria formação da identidade dos jovens e aumenta o seu comprometimento com a tribo. Isso é uma forma de se alcançar posições mais privilegiadas dentro da tribo. A tribo molda seus integrantes de maneira a levar a um certo nível de homogeneidade entre os indivíduos. Os valores dos jovens são os mesmos que permeiam as histórias dos mangás e que são oriundos da cultura japonesa.
\end{abstract}

Palavras-chave: Consumo. Tribos. Anime.

\section{ABSTRACT}

The present study is an ethnographic analysis of the tribe of animes, with the aim of unveiling the values, consumption practices, organization, behavior and culture of the tribe. We use video records, photos, and field notes to collect data in a participant observation research methodology. We conclude that the animes influence the visual styles, habits and consumption patterns of its members. The anime is the center of attention of young people. It is a kind of "totem" of the tribe. It is due to the consumption of anime that the tribe is formed. The group belong to the anime and the anime belongs to them. This occurs in a way that they become something within a whole, strengthening their identities and give meaning to their lives. Consumption within the tribe has a strong symbolic dimension. Having cultural products related to animes contributes to the very formation of the identity of young people and increases their commitment to the tribe. This is a way to achieve more privileged positions within the tribe. The tribe shapes its members so as to lead to a certain level of homogeneity among individuals. The values of the group are the same that permeate the stories of the manga and that come from the Japanese culture. Keywords: Consumption. Tribes. Anime.

\footnotetext{
Graduado em Marketing (Universidade de São Paulo/Brasil). E-mail: bruno.catao@ gmail.com.

${ }^{2}$ Doutora em Administração (Fundação Getúlio Vargas/Brasil). Professora na Universidade de São Paulo (São Paulo/Brasil). E-mail: claudiaraac@gmail.com.

${ }^{3}$ Graduado em Marketing (Universidade de São Paulo/Brasil). E-mail: eduonedu@ gmail.com.
} 


\section{INTRODUÇÃO}

Neste estudo trabalhamos a ligação entre os conhecimentos de consumo e os antropológicos, analisando o consumidor sob a perspectiva do grupo ao qual ele pertence. A ótica aplicada evidencia o pertencimento dos indivíduos aos grupos ou tribos, que são organizados em torno do consumo. O estudo se utilizou de eventos temáticos para aplicar a técnica da etnografia para estudar os costumes e relações dos jovens que apreciam animes.

Cabe antes explicar o elemento comum entre os jovens da tribo. Anime é o nome dado às animações (ou "desenhos animados") produzidos no Japão, e têm sua origem nos mangás, as histórias em quadrinhos produzidas no Japão. A influência ocidental no Japão do pós-guerra (década de 1950) foi responsável pelas primeiras produções de anime, que utilizaram o enquadramento cinematográfico para animar os desenhos dos mangás.

Os mangás são considerados como uma representação da cultura nipônica, uma vez que são produzidos e bastante difundidos por lá, carregando fortes traços culturais da cultura japonesa. Os animes consistem em similar representação cultural, como a adaptação animada do mangá. Assim, atingem a mesma tribo, sendo distintos somente em sua forma.

O foco de nossa pesquisa é a tribo de consumo de animes, também chamada de subcultura. Observamos e analisamos a comunidade que está inserida no ambiente dos animes, onde o referencial da cultura japonesa mostra-se fortemente presente. Mais especificamente, a pesquisa preocupa-se com a tribo de jovens que compartilha afinidade por animes no Brasil.

O objetivo geral do estudo é compreender a influência do anime na formação da tribo, bem como as influências da tribo nos valores, hábitos e padrões de consumo dos indivíduos que participam dela. Para isso o estudo busca analisar a força do anime como veículo carregado de conteúdo cultural, os padrões de consumo da tribo, sua composição e estrutura.

A justificativa do estudo está no fato de que a influência das tribos sobre os indivíduos faz com que eles tenham os mesmos hábitos, padrões de consumo, valores e dividam um mesmo universo simbólico cultural. Isso é resultado de uma cultura exterior que foi voluntariamente internalizada por cada um de seus membros. A partir de um consumo específico como o dos animes, os indivíduos organizam-se formando uma tribo. Dentro dela o comportamento dos indivíduos é característico e segue determinados padrões.

A influência do consumo na forma como as pessoas se organizam dentro de nossa sociedade é algo que não deve ser ignorado. Os referenciais culturais como formadores de tribo são um fenômeno crescente e cada vez mais comum na sociedade. É possível que isto esteja relacionado ao constante desenvolvimento de mídias e produtos culturais que experimentamos nas últimas décadas. O Estudo espera contribuir com o entendimento das relações entre cultura, valores e a influência de grupos nos hábitos de consumo de indivíduos pertencentes a tribos.

\section{REVISÃO DA LITERATURA}

Para os propósitos deste estudo, foi utilizada a definição de identidade dada por Hogg e Michell (1996). Os autores citam o modelo identity-world-view de Robbins (1973 apud HOGG; MICHELL), que postula a identidade como algo que abrange características pessoais e sociais como entendidas pela pessoa e pelos que a rodeiam. Os objetos de estudo deste trabalho buscam no consumo do anime e mangá a construção de uma identidade social aceitável dentro da tribo ou subcultura. O estudo de uma tribo só 
é possível a partir do momento em que há uma cultura que dá suporte aos seus integrantes. No caso desse estudo, a cultura que fornece esse suporte é a dos mangás, animes e a japonesa como um todo.

Gravett (2004 p.13) mostra que o termo mangá data do século XIX e foi usado pelo artista japonês Hokusai em 1814 para designar livros de "rascunhos excêntricos". O ideograma "man" tem um significado primário de "involuntário" e possui um significado secundário de "moralmente corrupto". O ideograma " $g a$ " significa imagens. O significado por trás do nome é condizente com o seu conteúdo extremamente amplo que aceita praticamente todo o tipo de temática, das mais adultas às mais infantis, das mais fúteis e simplificadas até as tramas psicológicas mais densas e complexas.

Até 1853, quando se deu o primeiro contato com os Estados Unidos e a consequente abertura econômica, o Japão esteve fechado às relações exteriores. O início das relações do Japão com outros países, visando a modernização do país durante a era Meiji (1868-1912) deu origem a slogans como Oitsuke! Oitsuke! (Alcançar! Superar!) para estimular as atividades econômica e cultural nacionais. Após a segunda guerra mundial, o Japão passou a seguir as regras do capitalismo americano, e as crenças de alcançar e superar fizeram-se valer também para o mangá.

A mentalidade insular dos japoneses provou ser capaz de absorver um conceito estrangeiro, adaptá-lo, desenvolvê-lo, e então exportá-lo de volta para o mundo. Com o mangá, os japoneses mostraram a mesma facilidade que tiveram com o automóvel, ou o chip de computador. Eles tomaram os fundamentos dos quadrinhos americanos - as relações entre imagem, cena e palavra- e, os fundiu a seu amor tradicional pela arte popular de entretenimento, os "niponizaram" de forma a criar um veículo narrativo com suas próprias características (GRAVETT, 2004, p.14).

O anime surgiu num período em que o Japão vivenciava a entrada de ideias ocidentais no país. Osamu Tezuka (1928-1989), reverenciado no Japão como o "Deus do Mangá", mostra em um de seus mangás autobiográficos as questões que o choque cultural trouxe para o seu trabalho: "Por que os filmes americanos são tão diferentes dos japoneses? Como eu posso desenhar quadrinhos que façam as pessoas rir, chorar e se emocionar como aquele filme? ". Após a segunda guerra mundial, a influência ocidental na criação dos animes se tornou forte, especialmente do cinema americano, o que facilitou sua difusão para o ocidente. Com o sucesso, surgiu um relacionamento quase simbiótico entre mangá e anime, que tem sustentado as duas indústrias desde então (GRAVETT, 2004, p.34).

No Brasil, a entrada dos animes ocorreu entre o fim dos anos 80 e o início dos anos 90. O anime foi exibido em redes de TV aberta e foi difundido com sucesso antes de que o mangá se desenvolvesse de forma expressiva no mercado brasileiro. $O$ crescimento da indústria dos mangás no Brasil ocorreu no final da década de 90 e início dos anos 2000, alavancado pelo sucesso obtido anteriormente pelo anime (FARIA, 2007; TANAKA, 2007).

Schouten e Mclaxander (1995) em seu estudo de subculturas de consumo, focalizam a subcultura de consumo orientada à Harley Davidson (Harley-Davidson-oriented subculture of consumption), com foco nos proprietários de motocicletas Harley-Davidson. Os pesquisadores apontam que o olhar crítico de um pesquisador sobre as subculturas de consumo deve ser direcionado às escolhas de consumo dos indivíduos e suas relações interpessoais: 
The most powerful organizing forces in modern life are the activities and associated interpersonal relationships that people undertake to give their lives meaning. In choosing how to spend their money and their time, people do not conform always or neatly to the ascribed analytic categories currently proffered by academia. They take part in the creation of their own categories. As consumer researchers we are uniquely positioned to identify and understand the organizing forces that people bring to their own lives through their consumption choices. In so doing we discover subcultures of consumption (SCHOUTEN; MCALEXANDER, 1995, p.43).

Os indivíduos se organizam em subculturas de consumo criando a sua própria categoria. Fazem isso de maneira consciente ou inconsciente para que suas vidas tenham um sentido. Para analisar o grupo ou rede formada pelos indivíduos os mesmos autores utilizaram a seguinte definição de subcultura:

We define a subculture of consumption as a distinctive subgroup of society that selfselects on the basis of a shared commitment to a particular product class, brand, or consumption activity. Other characteristics of a subculture of consumption include an identifiable, hierarchical social structure; a unique ethos, or set of shared beliefs and values, and unique jargons, rituals, and modes of symbolic expression (SCHOUTEN; MCALEXANDER, 1995, p.43).

Os jovens que apreciam o anime poderiam ser designados como uma subcultura de consumo, pois possuem uma estrutura social, partilham um conjunto de crenças e valores, possuem rituais e também jargões. Todavia, não há consenso de que a utilização da definição de subcultura seja a mais adequada. Bennett, critica em dois pontos a utilização do suporte teórico de subculturas:

In critically evaluating "subculture" as a valid framework for the sociological study of youth, music and style, I have identified two main issues. First, there is a problem of objectivity as a subculture is used in increasingly contradictory ways by sociological theorists. Secondly, given that in studies which use "subculture" in relation to youth, music and style there is a grounding belief that subcultures are subsets of society, or cultures within cultures, such a concept imposes lines of division and social categories which are very difficult to verify in empirical terms. Indeed, at the most fundamental level, there is very little evidence to suggest that even the most committed groups of youth stylists are in any way as "coherent" or "fixed" as the term "subculture" implies. On the contrary, it seems to me that so-called youth "subcultures" are prime examples of the unstable and shifting cultural affiliations which characterize late modern consumer-based societies (BENNETT, 1999, p.605).

Pensamos em tribos como algo menos rígido, mais fluido e associado a estilos de vida e aparência. Dessa maneira, foi escolhido o termo de tribo de consumo, ao invés de subcultura de consumo, para o grupo representado pelos jovens que apreciam anime. $\mathrm{O}$ anime teve o seu desenvolvimento num cenário pós-moderno. O cenário das sociedades contemporâneas baseadas no consumo parece mais dinâmico, portanto, mais relacionado às tribos do que às subculturas.

A tribo de consumo estudada é bastante dinâmica e complexa, por vezes combinando vários elementos culturais. As expressões de aparência e estilo da tribo são bastante fortes, mas há também a característica mutável das associações coletivas entre os indivíduos.

É importante lembrar que foi no cenário pós-guerra que surgiu o anime, o que situa a sua manifestação cultural em um contexto histórico de globalização e pós-modernidade. Este cenário tem como características maior fluidez e o fortalecimento das identidades dos indivíduos. Características estas que ressoam nas manifestações tribais.

Existe ainda o fator afetivo que guia os indivíduos, o qual podemos, de certo modo, pensar como uma comunhão dentro do cultural. Em seu trabalho de 2007, Faria afirma que o mangá é "eminentemente tribal, exatamente por exercer esse vínculo afetivo com os leitores, mesclando o imaginário individual 
com o coletivo, uma vez que a tribalização se dá em torno do afetivo, da necessidade da comunhão do estar junto".

A formação de tribos por indivíduos que compartilham as mesmas referências culturais torna possível um novo tipo de olhar sobre os mesmos. Os comportamentos individuais são substituídos pelos do grupo. Verifica-se que as ações das tribos são ditadas por um todo e não por cada um de seus componentes. Cova e Dalli (2008 p.16) chamam o desejo da ação do grupo de "nós-intenção" (weintention), "Nós-intenção é o desejo do grupo como um todo. Essa comunidade pode ser considerada como uma única cabeça com muitos neurônios representados pelos seus membros". A nós-intenção é considerada como a vontade do grupo. As ações dos indivíduos orientados por ela reforçam simultaneamente os valores comuns da tribo e o pertencimento do indivíduo ao grupo, dado que sua ação individual tem correspondência com a vontade coletiva.

As ações dos indivíduos guiadas pelo desejo do grupo como um todo só são possíveis pelo fato de que os integrantes desses grupos compartilham de um universo simbólico fornecido pelos meios de comunicação. Para Maffesoli (1988), os meios de comunicação como a televisão e a internet fornecem imagens remetendo às "figuras do estilo comunitário" que geram modos de comunidade e a tribalização.

Tribos que compartilham padrões de ações e se organizam em torno dos meios de comunicação merecem um olhar crítico, pois possuem comportamentos específicos de consumo de acordo com seus valores, cultura e hábitos. Ostergaard e Jantzen (2000) afirmam que o simbolismo do produto consumido cria um universo para a tribo.

Sabe-se que os integrantes da tribo se organizam seguindo um referencial, pertencem a um grupo, assim como o grupo pertence a eles. Deve-se esclarecer que os indivíduos pertencentes não são todos idênticos, nem agem somente de acordo com a intenção do grupo. Faz-se necessário compreender que existem diferentes níveis de comprometimento dos integrantes da tribo com seus referenciais, seus ideais e valores. O que não significa que todos os seus indivíduos ajam somente de acordo com a intenção da tribo ou ainda de acordo com os seus ideais que seriam de algum modo idênticos aos da tribo. Ao invés disso, dentro das tribos existe uma estratificação entre seus membros. Os membros podem ser classificados de acordo com a sua presença ou ausência na tribo e quanto à intensidade de seu comprometimento com o referencial cultural (MAFFESOLI, 2005).

Em um estudo sobre os punks, Fox (1987) os classifica em Hardcore, Softcore, Preppie e Spectators, os grupos interagem uns com os outros e possuem diferentes posições a respeito de seus papéis dentro da tribo. Na tribo dos jovens que apreciam anime, classificações hierárquicas também fazem sentido. É possível diferenciar os seus integrantes de acordo com o seu comprometimento com o referencial da cultura dos animes. Nosso estudo relaciona cultura, consumo e a composição da tribo analisada. Convidamos os leitores a utilizarem-se da literatura previamente discutida como lente para visualizar a tribo do anime, nosso objeto de estudo.

\section{METODOLOGIA}

A tribo de jovens que apreciam anime é bastante engajada, o que possibilita a realização de eventos de grande magnitude. Participam bandas nacionais e internacionais (por vezes as mesmas que tocam nas trilhas sonoras dos animes), palestrantes - tanto brasileiros como estrangeiros - e atrações variadas relacionadas à temática do evento. As convenções de animes são frequentados por jovens de todo o Brasil e, em alguns casos, também do exterior. Nos eventos em que a observação da tribo foi realizada (de 2009 a 2015), o público variou entre 25.000 e 70.000 . 
A pesquisa foi realizada em todas as edições de quatro eventos anuais de animes realizados em 2009, 2010, 2011, 2012, 2013, 2014, 2015 na cidade de São Paulo. Um dos pesquisadores permaneceu em campo durante todos os dias dos eventos registrando as suas experiências e observações utilizando diários de campo, fotografias e filmagens características do método etnográfico.

A pesquisa realizada foi de caráter qualitativo, utilizando o método de estudo etnográfico orientado ao mercado (market-oriented ethnogaphy). Segundo Arnould e Wallendorf (1994 p.484), "The term market-oriented ethnography refers to an ethnographic focus on the behavior of people constituting a market for a product or service". No contexto do presente estudo, os indivíduos constituem o mercado para o produto cultural anime e seus produtos relacionados.

O método foi escolhido pela sua adequação ao estudo de tribos devido à amplitude de informações que ele permite coletar. De fato, a etnografia foi escolhida e utilizada para que a tribo de consumo fosse analisada com a profundidade e amplitude necessária para entender seus valores, hábitos e padrões de consumo. O método, por não ser altamente estruturado, possibilita aos pesquisadores descobertas que eram inesperadas antes da entrada em campo (MALINOWSKI, 1984).

Um dos pesquisadores antes de ir a campo já possuía uma certa familiaridade com os jovens consumidores de anime, dado que assistiu a algumas séries, comprou mangás e conviveu com amigos que poderiam ser classificados como softcore da tribo. Isso permitiu ao pesquisador ter algum conhecimento prévio sobre jargões e consumo dos integrantes da tribo. Tanto a aparência deste pesquisador quanto a idade, o estilo de vida e os valores eram similares aos dos integrantes da tribo e tal fato contribuiu para que houvesse acesso mais fácil aos objetos de estudo.

Foram utilizados diferentes graus de participação do pesquisador, aproximando-se de um insider (membro da tribo) para ter acesso ao "backstage", e, em outros momentos aproximando-se de um outsider (não-membro) na tentativa de reduzir o viés da presença do pesquisador (ARNOULD; WALLENDORF, 1994).

As técnicas utilizadas foram de observação participativa e de entrevistas não estruturadas com integrantes da tribo. Os dados de campo foram registrados na forma de diários de campo, registro visual fotográfico (Foto-etnografia) ou registro utilizando-se filmagens na (Vídeo-etnografia).

A primeira experiência de campo dos pesquisadores ocorreu em um evento que possuía a duração de sete dias; as demais tiveram duração de quatro dias cada. Os dados eram coletados ao longo do dia, iniciando pela manhã e terminando ao anoitecer. As visitas repetidas a campo tiveram o propósito de atingir com maior segurança a saturação do conhecimento sobre a tribo. Durante três dos dias em que um dos pesquisadores foi ao evento, foi tal como um insider, utilizando indumentárias cosplay, o que permitiu ao pesquisador ter acesso a diferentes níveis da tribo, compreendendo melhor a experiência vivenciada por um insider. É importante citar que a existência da pesquisa e seus objetivos não foram revelados aos indivíduos presentes no evento durante o estudo.

Com o uso da técnica etnográfica nas intervenções foram realizadas mais de seiscentas fotos de integrantes da tribo, de seus referenciais culturais, suas atividades, estilos visuais de vestimentas, produtos consumidos, entre outros tópicos que interessam ao estudo. Produzimos também algumas horas de gravação dos jovens no espaço do evento, incluindo entrevistas com representantes de grupos da tribo. 


\section{ANÁLISE DOS RESULTADOS}

\section{A Tribo}

A tribo dos indivíduos que consomem animes tem variações maiores do que as esperadas em sua composição. Os animes não atraem apenas jovens, mas várias faixas etárias. Muitos adultos e crianças (junto com seus pais) participam do evento e fazem parte da tribo.

A tribo é complexa e existem diferenças entre seus integrantes que se organizam em grupos dentro dela. Uma das bases da diferença desses grupos parece estar no visual, visto que apresentam diversas variações.

Foram propostas pelos pesquisadores algumas classificações para os grupos presentes nas tribos baseadas nas diferenças visuais, comportamentais e de valores que os integrantes apresentam entre si em busca de facilitar o estudo da tribo.

As classificações não são absolutas e ao longo das experiências de campo foram aprimoradas para obter maior precisão. As categorias são reconhecidas pelos próprios integrantes da tribo analisada, e podem ser resumidas da seguinte forma:

- Cosplayers: utilizam vestimentas que procuram ser idênticas às dos personagens da mídia e tentam reproduzir até mesmo seu comportamento, em uma tentativa de incorporar o personagem.

- Gamers: Possuem intimidade com jogos eletrônicos e os jogam com frequência.

- Otakus: Possuem amplo conhecimento sobre a cultura dos animes e possuem comportamentos considerados excêntricos. Seu visual ostenta uma mistura desordenada de elementos variados que pertencem ao universo cultural do anime. São fãs que conhecem com abrangência as séries e as bandas da tribo.

- Lolitas: Garotas que se vestem como bonecas seguindo estilos específicos de vestimentas e personalidade.

- Colecionadores: Participam de reuniões e de salas de exposição para onde levam seus artigos de coleção para ficarem juntos aos artigos de outros aficionados. Alguns dos objetos colecionáveis observados foram: bonecas, figuras, réplicas de armamentos, CDs, DVDs, mangás e artesanatos.

- Medievais: Indivíduos que apreciam vestimentas, acessórios, armamentos e cultura de temática medieval. Em alguns casos confeccionam suas próprias vestimentas, alimentos e armamentos.

- Yuri-Yaoi: Apreciadores de Animes de gênero homossexual

- JK-Music: Apreciadores de bandas japonesas e coreanas. Utilizam vestimentas típicas de integrantes de bandas ou grupos musicais. Eventualmente podem até mesmo participar de bandas e montar grupos de dança com coreografias específicas.

- Cosfighters: Apreciadores de animes que praticam artes marciais e reproduzem movimentações acrobáticas de animes. Entre eles as batalhas campais e wrestling são muito populares.

- Tokusatsus: Apreciadores de séries de super-heróis japonesas como Changeman e PowerRangers.

- Furries: Apreciadores de séries que possuem animais como protagonistas. Criadores de personagens antropozoomórficos, dos quais podem resultar histórias ou vestimentas.

- Fanzineiros: Jovens que são produtores de seus próprios mangás, fazendo suas publicações e podendo até mesmo fabricar e vender materiais relacionados às suas histórias (como adesivos e pelúcias).

Existem ainda outras classificações possíveis, especialmente porque os gêneros de mangá e anime são muito variados, abrangendo temáticas como esporte e terror, ou voltados a públicos específicos, 
como feminino ou masculino. Entretanto, não foram encontrados em números relevantes os membros de tais denominações, fazendo com que os pesquisadores se ativessem às citadas acima.

A existência dos grupos dissimilares entre si levantaria um questionamento sobre seu pertencimento à mesma tribo, no entanto o que deve ser analisado é o quão diferente eles são entre si. A título de exemplificar que suas diferenças não são tão grandes assim focaremos os $J K$-Music e Gamers.

É verdade que jovens que apreciam o $J$-rock e o $K$-pop são diferentes dos que apreciam os jogos, no entanto, há algo que os une e que não deve ser negligenciado. Existem animes de gênero musical, retratando histórias de bandas jovens se desenvolvendo. As bandas mais famosas, usualmente são convidadas para tocar na abertura e ao final dos episódios de anime. Os J-rocks estão ligados aos animes, sendo que em alguns dos casos o conhecimento das bandas japonesas é posterior à descoberta das séries de animes pelos integrantes da tribo.

Nos Gamers, por sua vez, existe uma relação estreita entre os animes e os games, muitos dos jogos de sucesso dão origem a animes e muitos dos animes de sucesso dão origem a jogos, em uma espécie de relação simbiótica. Títulos de jogos são também publicados em formatos de mangá em alguns casos e a temática dos animes e cultura japonesa é presente em ambos.

Dessa maneira cada um dos grupos que foram descritos acima tem essa ligação aos animes e à sua cultura. Existe uma espécie de escolha de tema dentro do referencial cultural que faz com que eles apresentem também diferenças entre si, no entanto, o conhecimento sobre o universo dos animes nos integrantes de diferentes grupos é similar e cada um deles consegue reconhecer os outros indivíduos. Mesmo dentro do que podemos definir como sendo diferentes grupos da tribo, os jovens possuem consumo e valores muito similares entre si.

Os grupos interagem entre si, compreendem uns aos outros e estão todos relacionados aos animes. Isso faz com que a soma dos grupos resulte na tribo de consumo dos jovens que apreciam animes. Mesmo que os grupos apresentem entre si essas diferenças, o que se vê é que seus padrões de consumo e valores são muito similares, modificando apenas a temática dentro do referencial cultural dos animes.

Existe mais um nível dentro da tribo a ser analisado: o da hierarquia. Utilizaremos uma hierarquia similar a proposta por Fox (1987). Para fins de classificação e estudo, os jovens foram divididos como pertencentes aos seguintes grupos hierárquicos: Hardcore, Softcore, Casuais e Expectadores.

Os Hardcore são os membros mais envolvidos com a cultura dos animes. São os jovens que mais possuem prestígio dentro da tribo, responsáveis por ditar os padrões e as tendências para os outros indivíduos. São altamente comprometidos e têm seus estilos bastante influenciados pelos animes.

Os Softcore são membros um pouco menos comprometidos com os ideais da cultura da tribo, o seu conhecimento da cultura é menor do que o dos Hardcore. Apesar de certa resistência a internalizar os valores da tribo, o seu envolvimento com os animes e a tribo ainda é elevado quando comparado ao do público geral.

Os Casuais copiam o estilo visual dos outros integrantes mais envolvidos na tribo, ou agem de forma similar, tendo, no entanto, um envolvimento com a tribo apenas superficial. O seu conhecimento da cultura é baixo, bem como seu comprometimento com animes e a tribo. A sua participação nos eventos é pontual. É apenas uma experiência casual (descompromissada) para eles.

Os Expectadores, são aqueles que não pertencem à tribo. São outsiders, que têm interesse em observar a tribo como forma de entretenimento ou outro interesse.

Na tribo, o número de indivíduos hardcore é menor do que o de softcore que por sua vez é menor do que o de casuais que são menos numerosos que os expectadores, ou seja, existem menos indivíduos nos níveis em que maior comprometimento é necessário. 
Essas classificações hierárquicas são interessantes para o estudo, pois acrescentam uma nova dimensão à análise da tribo e de seus grupos. A dimensão dos níveis de comprometimento, segundo as quatro categorias citadas, nos traz maior precisão de análise. Indivíduos de diferentes níveis de comprometimento podem ser identificados nos grupos da tribo. Isso torna-se mais claro com a observação de suas interações durante os eventos. Como forma de mostrar a influência da hierarquia proposta em quatro níveis, será descrito o que foi evidenciado no grupo de cosplayers, embora o mesmo possa ser evidenciado em cada um dos outros grupos analisados.

Os cosplayers hardcore são o centro das atenções no evento de animes. Suas vestimentas são de alta qualidade de manufatura e muito similares às vestimentas vistas nas personagens propriamente ditas. Possuem um conhecimento detalhado dos personagens e da trama da série de anime que acompanham. Para os hardcore, cosplay não é apenas um estilo visual, muito menos uma fantasia. Cosplay é um processo pelo qual se incorpora (termo que foi utilizado por um cosplayer em uma das entrevistas realizadas no estudo) um personagem, suas ações e até mesmo seus aspectos psicológicos. Isso é feito como uma forma de demonstrar admiração e identificação do cosplayer com a personagem que ele homenageia.

Durante o dia esses cosplayers são alvo de centenas de fotos, alguns deles participam de competições de cosplay oficiais ou amadoras e agem como se fossem realmente os próprios personagens. Percebe-se que para os cosplayers hardcore, não existe apenas uma preocupação com o estilo, mas com uma maneira de pensar e agir, valores específicos relacionados à cultura dos animes que permitem que esse processo de incorporação do personagem aconteça. Esses jovens eventualmente modificam a cor dos olhos com lentes de contato, pintam e arrumam seus cabelos para representar o personagem. Em alguns casos, fabricam réplicas de equipamentos utilizados pelas personagens, tudo isso num esforço para se aproximar ao máximo do visual da fonte.

O comprometimento desses jovens com a tribo e sua cultura é bastante elevado, chegando até a haver um certo grau de sacrifício. As vestimentas dos cosplayers são usualmente desconfortáveis devido ao grande número de detalhes compondo o visual. Os armamentos podem ser grandes e pesados dificultando a mobilidade. $\mathrm{O}$ fato de se tornarem o centro das atenções faz com que se tenha a liberdade sacrificada devido ao número de fãs da personagem que desejam se comunicar com quem está incorporando seu ídolo. Além disso, as pessoas que gostaram da vestimenta tiram fotos e querem conversar para mostrar suas expressões de reconhecimento à indumentária cosplay. Outro ponto a ressaltar é que os custos da confecção das vestimentas são elevados. Ao questionar um cosplayer hardcore sobre quanto ele havia gasto compondo o visual, ele respondeu que lhe havia custado aproximadamente $\mathrm{R} \$ 1.500,00$.

$\mathrm{Na}$ esfera do cosplay em específico foi evidenciado que as fotos representam uma expressão de reconhecimento do cosplayer pelo fotógrafo. Os cosplayers hardcore dificilmente tirarão fotos de outros cosplayers. Os hardcore reconhecem os softcore como integrantes da tribo e possuem atitudes positivas em relação a eles. Em alguns casos dão dicas de como melhorar o seu visual, incentivam, e elogiam os cosplayer softcore. Além disso, não se importam de tirar fotos juntamente a esses cosplayers. É provável que os hardcore, encarem os softcore como membros em transição, dessa forma incentivando o seu desenvolvimento.

Os casuais, no entanto, são vistos com outros olhos pelos cosplayers, que os percebem como indivíduos que não conseguem fazer o cosplay de maneira adequada, pois não conhecem seus personagens a fundo e têm indumentárias de baixa qualidade. Existem inclusive jargões característicos dos hardcore utilizados para se referir aos casuais de maneira pejorativa como o termo "cos-pobre" (ou ainda tosco-play). A presença dos expectadores é ignorada pelos hardcore. 
Os cosplayers softcore ocupam uma posição intermediária na tribo, possuindo menos prestígio que os hardcore, mas ainda assim sendo admirados. A qualidade de suas vestimentas é boa, mas encontra limitações como a falta de experiência do cosplayer ou resistência a modificar o visual de alguma maneira mais característica como o fazem os hardcore. Os softcore possuem um bom conhecimento da cultura dos animes, e razoável sobre o personagem que estão interpretando, apesar de ser considerado limitado quando comparado ao de um hardcore. Ao contrário do hardcore, o softcore não está tão preocupado com a perfeição da indumentária e a incorporação do personagem. Ele irá procurar um visual tão próximo quanto possível, e utilizar seus conhecimentos sobre a personagem para agir em alguns momentos de maneira similar a ele.

Em relação às fotos, os cosplayer softcore são mais ativos, procuram tirar fotos de cosplayers hardcore, pelos quais possuem grande admiração, e também tiram fotos de outros cosplayers softcore. Para o softcore, os "verdadeiros cosplayers" são os hardcore, o que pode ser notado quando eles assistem com bastante admiração as suas apresentações e competições. Os softcore não possuem admiração pelos casuais, pois sob sua percepção não são cosplayers propriamente ditos. Os expectadores são percebidos pelos softcore como pessoas simpáticas ao evento.

Os cosplayers casuais ocupam uma posição periférica na tribo. A qualidade de suas vestimentas é geralmente baixa, dado que eles não estão muito preocupados com sua qualidade ou não conhecem o personagem de maneira aprofundada. O conhecimento que os casuais possuem da cultura é somente superficial. Fazem cosplay como atividade lúdica ou numa tentativa de pertencer ao grupo dos softcore ou hardcore, no que falham por falta de comprometimento com o anime e pelo seu status de casual.

Para os casuais pertencer à tribo é mais uma questão de estilo do que de cultura e comprometimento. É uma espécie de diversão, que toma lugar pontual ou casualmente nos eventos de animes que resolvem frequentar.

Os casuais demonstram grande interesse em tirar fotos dos hardcore e os softcore, mas poucos integrantes da tribo tiram fotos deles. Muitas vezes, quando ocorre de tirarem fotos dos casuais, os integrantes da tribo têm a intenção de zombar do cosplay de baixa qualidade. Muitas vezes os próprios casuais fazem suas indumentárias com um intuito de zombaria, e assim já sabem qual é o propósito das fotos.

Os casuais admiram os hardcore e os softcore. Interagem também com os expectadores, o que pode ser justificado pelo fato de que eles se encontram nos limites do que é definido e aceito como membro pela tribo.

Expectadores: também chamados outsiders, esses indivíduos não pertencem à tribo, no entanto frequentam os eventos por algum interesse. Os expectadores não têm comprometimento com a tribo e tem baixo ou nenhum conhecimento sobre a cultura da tribo. Fotografam os cosplayers ou outras coisas que encontrarem de interessante. Normalmente eles não sabem o que são os cosplayers a fundo, nem os admiram, apenas estão impressionados com aspectos visuais como armamentos de grandes proporções, cosplayers atraentes, ou grandes grupos de cosplayers.

Para os expectadores, a tribo, a sua estética, ação e organização é uma espécie de atração do evento. Eles freqüentam os eventos como forma de ver a tribo, da qual eventualmente alguns deles poderão acabar vindo a se tornar parte. No momento que são expectadores, no entanto, eles possuem acesso apenas superficial aos indivíduos participantes da tribo. Os expectadores, são de uma certa maneira importantes para a tribo, pois servem como oposição à identidade da tribo. Cada integrante da tribo é diferente de quem não pertence à tribo. Sem os indivíduos que não pertencem à tribo, a tribo não seria algo único ou diferente dos demais, essa oposição serve para fortalecer a percepção de pertencimento dos integrantes da tribo e fortalece a sua identidade. 
A classificação breve dos cosplayers segundo a hierarquia explicada não tem como objetivo mostrar todas as relações entre os hardcore, softcore, casuais e expectadores dentro do grupo de cosplayers. O objetivo é mostrar como se dão as interações entre os níveis hierárquicos das categorias, sabendo que elas são as mesmas que ocorrem entre as demais denominações da tribo. Dessa forma, os gamers, são separados com base no conhecimento e habilidades dos jogadores, as lolitas com base no estilo de ser lolita, visual e conhecimento, os tokusatsus com itens colecionáveis relativos a séries de super-heróis e assim por diante em cada um dos grupos que formam a tribo. Essas relações estão presentes em toda a tribo, resultando na admiração de alguns de seus integrantes pelos outros a partir das posições que eles ocupam dentro da tribo. O que importa é o comprometimento do indivíduo com o referencial cultural.

Sob essa perspectiva, ainda pode-se notar que os hardcore dentro da tribo possuem um papel importante dentro dos eventos, reunindo os outros integrantes e até mesmo servindo como atração para os outros níveis hierárquicos. O uso dessa hierarquia é importante para a compreensão da tribo. A observação de valores, padrões de consumo e características intrínsecas da tribo, faz-se mais precisa nos indivíduos hardcore e softcore.

\section{O evento}

No decorrer do evento, os jovens permanecem a maior parte do tempo com grupos de amigos, e algumas vezes cantam músicas das aberturas dos animes juntos e costumam vestir-se com a mesma temática. $\mathrm{O}$ canto em conjunto, em grupos de amigos remete a uma experiência eminentemente tribal. Como uma espécie de cerimônia ou ritual dentro da tribo.

Seguindo a tradição dos mangás, nos quais os personagens se comunicam através de balões com frases escritas, ocorre uma forma de comunicação típica de eventos de anime. Os participantes do evento podem muitas vezes serem vistos carregando pequenas placas nas quais escrevem frases com marcadores, variando desde de jargões da tribo, citações de séries, anúncios e quaisquer outras mensagens que o portador da placa ache interessante transmitir.

Roupas também são muito importantes para os participantes do evento, mesmo quando não estão ligadas a cosplay. Como os participantes utilizam camisetas ou acessórios de suas séries, animes, filmes favoritos, os trajes muitas vezes indicam os interesses das pessoas, facilitando a aproximação daqueles com interesses similares.

Os visuais utilizados pelos adolescentes no geral são muito carregados. Utilizam bottons, jeans, cores escuras, cabelos pintados e acessórios relacionados aos animes. O chamado J-Look, o japanese look, é muito apreciado entre os jovens.

No geral os integrantes da tribo gostam de fazer performances e de ser notados. Para isso cantam, desfilam, tiram fotos e se vestem de maneira chamativa. Essas ações são formas de fortalecer a identidade da tribo e de expressar seus desejos de serem reconhecidos.

Em relação aos valores percebe-se que a sexualidade possui um peso muito grande, o que pode ser visto pelas vestimentas, comunicação nas plaquinhas e atrações do evento. Tão forte quanto a sexualidade é a apreciação da violência. Os jovens não são violentos uns com os outros, ao contrário, se relacionam muito bem, mas apreciam assistir a cenas de violência, armamentos e lutas. Essas questões relacionadas à violência e sexualidade mostram-se ligadas ao referencial cultural japonês, pois nos mangás e animes voltados ao público adulto, o sexo e a violência não têm limites.

Um exemplo da força da cultura japonesa dentro da tribo é a assimilação do idioma entre os jovens de maior comprometimento. Mesmo quando não falam japonês, muitos deles usam expressões da língua e até cantam músicas inteiras no idioma, contanto que façam parte da trilha sonora de algum anime ou jogo. 
Apesar da maioria dos integrantes da tribo não ser de origem nipônica, a tribo está organizada em torno do universo cultural oriental. Tal fato fortalece a construção da identidade do grupo. Vemos esta escolha do indivíduo como uma forma de auto-expressão e de dar significado à sua existência. Não é necessário que o indivíduo tenha um envolvimento prévio ou ligação com a cultura, pois isso é desenvolvido ao longo do processo em que ele se insere na tribo passando de outsider para insider (ascendendo nos níveis de comprometimento).

A facilidade de lidar com diferenças entre os integrantes da tribo pode ser facilmente notada nos sujeitos da pesquisa. Há uma aparente ausência de discriminação dos membros em relação aos outros membros que pertençam a minorias, ou que possuam necessidades especiais dentro da tribo. Essa ocorrência dentro da estrutura social seria justificada pelo fato de que os indivíduos estão reconhecendo como prioridade as suas posições ocupadas dentro da tribo e não suas outras posições sociais. Como apresentado por Schouten e Macalexander (1995) em seu estudo,

[...] em nossa cultura do consumidor as pessoas não se definem de acordo com constructos sociológicos. Elas o fazem em termos de atividades, objetos, e relações que dão sentido às suas vidas. São esses objetos e bens de consumos sobretudo que, que dão suporte ao seu lugar no mundo social (SCHOUTEN; MCALEXANDER, 1995, p.47).

Assim, um integrante da tribo é um integrante da tribo antes de qualquer outra classificação social a que ele pertença.

Os jovens aparentam carência afetiva e necessidade de chamar a atenção, sendo que, a própria sensação de pertencimento à tribo talvez possa ser uma tentativa de amenizar esse sentimento. Isso adequa-se ao fato de que as tribos se organizam em torno do afetivo.

Por fim, analisando o consumo dentro do espaço dos eventos evidenciam-se alguns consumos característicos da tribo. Os integrantes dão elevada atenção aos estandes de mangás, onde compram as histórias em quadrinhos para leitura e coleção. Formam-se filas bastante grandes apenas para entrar nos estandes. Uma coleção inteira de mangá, com a história completa de uma das séries, pode ter algumas centenas de volumes. Os jovens, emocionalmente envolvidos com a compra, podem comprar dezenas de mangás de uma só vez.

Outro produto muito consumido nestes eventos é o Mupy, uma bebida não alcoólica à base de soja, que por questões de preço e sabor se tornou a bebida oficial destas ocasiões. A própria atitude positiva do jovem em relação à bebida fortalece o seu envolvimento com a cultura da tribo.

Existem também as figures, que são miniaturas das personagens dos animes que fazem sucesso entre os jovens. As figures podem possuir em sua temática uma forte sexualidade, presença de armamentos ou representar cenas icônicas do universo dos animes.

Além dos exemplos de consumo citados que estão presentes em grande intensidade na tribo, existem muitos produtos específicos consumidos pelos jovens, como vestimentas (cosplay, lolitas, furry), réplicas de armamentos, toucas com temática de anime, "card games" entre outros.

No estudo da subcultura de proprietários de Harley-Davidson Schouten \& Macalexander (1995) explicam o sentido do acúmulo de posses especializadas relacionadas à cultura:

The biker accumulates especialized possessions that both demonstrate and increase commitment to motorcycling and to the Harlyu-Davidson brand. Material side bets include branded leathers and other clothing, expensive accessories for the motorcycle, tattoos, and the virtual shrines erected by many bikers for the purpose of storing, maintaining, and displaying their bikes (SCHOUTEN; MCALEXANDER, 1995, p.56). 
De maneira similar ao ocorrido com os proprietários de Harley-Davidson, as compras dos integrantes da tribo visam demonstrar e aumentar o comprometimento de seus integrantes com o referencial dos animes, o que é um meio pelo qual o pertencente da tribo pode acessar níveis hierárquicos mais elevados na mesma, como os hardcore e softcore. O acesso a esses níveis hierárquicos, por sua vez, é desejado pelos integrantes que almejam obter o reconhecimento dos outros integrantes e ocupar posições mais "privilegiadas" dentro da tribo. Isso esclarece o papel representado pelo consumo e a sua importância para a formação e manutenção da tribo e de sua estrutura.

\section{CONCLUSÃO}

O objetivo da pesquisa foi analisar a influência do anime na formação da tribo, bem como as influências da tribo nos valores, hábitos e padrões de consumo dos indivíduos que participam dela.

A força do anime como veículo carregado de conteúdo informacional e cultural pode ser evidenciada pela complexidade do anime como referencial cultural. $\mathrm{O}$ anime traz em si a cultura japonesa e muitos de seus valores. Possui ainda uma herança do cenário de sua criação, da pósmodernidade, do pós-segunda guerra (das cicatrizes deixadas no Japão) e do processo pelo qual o Japão se transformou sob a influência do capitalismo americano e da cultura do cinema ocidental. $\mathrm{O}$ anime tem grande apelo entre os jovens por sua expressividade visual e temática variada que aborda inclusive temas mais "restritos", como a violência e o sexo.

Fica claro que a formação da tribo só é possível devido à existência dos animes. Estes influenciam os estilos visuais, hábitos e padrões de consumo dos integrantes. $\mathrm{O}$ anime é o centro da atenção dos jovens. É uma espécie de "totem” da tribo de consumo. É devido ao consumo do anime que a tribo se forma. Os jovens pertencem ao anime e os anime lhes pertence. Isso ocorre de maneira que eles se tornam algo dentro de um todo, fortalecendo as suas identidades e conferindo sentido às suas vidas.

O consumo dentro da tribo possui forte dimensão simbólica. Exemplo disso são as plaquinhas de madeira, compradas no evento e utilizadas pelos jovens. Essas placas têm elevado poder de comunicação entre os membros da tribo. A sua posse está relacionada à identificação de seu proprietário como membro da tribo e além disso podem até mesmo ser uma aproximação dos jovens. Além disso, o uso das placas os aproxima dos personagens que falam por meio de "balões". Os produtos são comprados pelos integrantes da tribo por terem um significado dentro da cultura dos animes. Possuir produtos culturais relacionados aos animes contribui para a própria formação da identidade dos jovens e aumenta o seu comprometimento com a tribo. Isso é uma forma de se alcançar posições mais privilegiadas dentro da tribo.

A influência da tribo nos valores, hábitos e padrões de consumo mostra-se bastante aparente. A tribo molda seus integrantes de maneira a levar a um certo nível de homogeneidade entre os indivíduos. Os valores dos jovens da tribo são os mesmos que permeiam as histórias dos mangás e que são oriundos da cultura japonesa. Um exemplo de valor cultural japonês apropriado dos mangás é o espírito de competição entre os membros, que pode ser reconhecido em várias dimensões: na competição da melhor vestimenta de cosplay, das lutas, nos jogos, na intenção de completar coleções de mangás e figures.

A tribo é ampla possuindo todo um referencial cultural que orienta seus indivíduos. Os seus integrantes possuem diferentes níveis de comprometimento e foram classificados segundo essa dimensão em hardcore, softcore, casuais e expectadores. Esta classificação diz respeito ao envolvimento com a tribo e seu referencial cultural e mostra que os indivíduos ocupam posições centrais ou periféricas dentro da organização da tribo. 
O estudo traz como implicações práticas para gestores o fato de que ao interagir com a tribo utilizando comunicação ou oferecendo produtos, deve-se estar ciente de que a tribo não é totalmente homogênea. Assim, o direcionamento pode ser feito a algum dos grupos da tribo ou utilizar-se dos pontos comuns entre todos eles para o direcionamento à tribo como um todo. Conhecer valores e a composição de uma tribo é relevante também para o desenvolvimento de novos produtos, ou para uma segmentação de mercado.

\section{REFERÊNCIAS}

ARNOULD, E. J.; WALLENDORF, M. Market-Oriented Ethnography: Interpretation Building Marketing Strategy Formulation, Journal of Marketing Research, v. 23, 1994.

BELK, R. W.; KOZINETS, R. V. Videography in marketing and consumer research. Qualitative Market Research: An international Journal, v.8, 2005.

BENNETT, A. Subcultures or Neo-Tribes? Rethinking the Relationship Between Youth, Style and Musical Taste. Sociology, v.33, n.3, University of Kent NY, 1999.

CORTÉS, TANIA ARCE; Subcultura, contracultura, tribus urbanas y culturas juveniles: ¿homogenización o diferenciación? Revista argentina de sociologia, v.6, n.11, Buenos Aires jul./dic. 2008.

COVA, B., DALLI, D. From communal resistance to tribal value creation. Euromed Marseille \& Università Bocconi Milan, 2008.

COVA, B.; COVA, V. Tribal Marketing: The Tribalisation of Society and its Impact on the Conduct of Marketing. European Journal of Marketing, 2002.

EVERARDO. Perspectivas do Método Etnográfico em Marketing: Consumo, Comunicação e Netnografia. Anais... ENANPAD, 2005.

FARIA, M. L. Comunicação Pós-Moderna Nas imagens dos Mangás. Biblioteca Ir. José Otão Pontifícia Universidade Católica do Rio Grande do Sul, 2007.

FOX, K. J. Real Punks and Pretenders: The Social Organizatiom of a Counterculture. Journal of Contemporary Ethnography, v.16, 1987.

GRAVETT, P. Manga, sixty years of japanese comics. Conrad editora do Brasil, São Paulo, 2004.

HOGG, Margaret K.; MICHELL, PAUL C.N., Identity, Self and Consumption: A Conceptual Framework. The Dryden Press, Dryden, 1996.

KASSARJIAN, H. H. Content Analysis in Consumer Research. The Journal of Consumer Research, v. 4, 1977.

MAFFESOLI, M. A ética da estética: Homo Estheticus. Revista de Comunicação \& Linguagens NT, 1988.

MAFFESOLI, M. Comunidade de Destino. Horizontes Antropológicos, ano 12, n.25, Université Paris V, 2005.

MALINOWSKI, B. Argonauts of the Western Pacific, An Account of Native Enterprise and Adventure in the archipelagoes of melanesian New Guinea. São Paulo: Abril Cultural, 1984. 
MCCRACKEN, G. Clothing as language: an object lesson in the study of the expressive properties of material culture. In: REYNOLDS, B; STOTT, M. (Eds.). Material Anthropology: Contemporary Approaches to Material Culture. New York: University Press of America, no prelo, 1986.

MCCRACKEN, G. Culture and Consumption. New Approaches to the symbolic character of consumer goods and activities. Bloomington and Indianapolis: Indiana University Press, 1990.

OSTERGAARD, P.; JANTZEN, C. Shifting Perspectives in Consumer Research: From Buyer Behaviour to Consumption Studies. CBS Press, Copenhagen, 2000.

SCHOUTEN, J. W.; MCALEXANDER, J. H., Subcultures of Consumption: An Ethnography of the New Bikers. Journal of Consumer Research, v. 22, 1995.

TANAKA, N. D. O Mangá Como Material Alternativo no Ensino de Japonês Como Língua Estrangeira em Nível de Graduação. Universidade do Estado do Rio de Janeiro, dissertação de doutorado, 2007. 